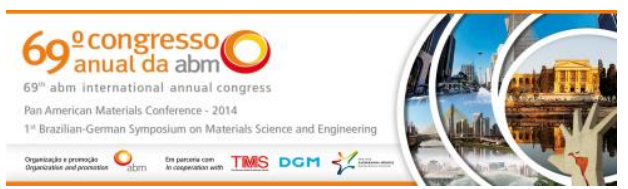

Tema: Iniciação Científico-Tecnológica

\title{
ANÁLISE DA INFLUÊNCIA DE PARÂMETROS DE ESCÓRIAS DE REFINO SECUNDÁRIO E DE PROCESSO NA LIMPEZA INCLUSIONÁRIA DE AÇOS*
}

\section{Resumo}

\author{
Franciele de Almeida Silva ${ }^{1}$ \\ Wagner Viana Bielefeldt ${ }^{2}$ \\ Antônio Cezar Faria Vilela ${ }^{3}$
}

A escória utilizada no refino secundário deve possuir propriedades adequadas para que auxilie no controle inclusionário dos aços. Tendo isto em vista, há vários parâmetros que devem ser levados em conta para se obter uma escória ótima que irá auxiliar na limpeza dos aços. Como uma técnica de medição, o valor de oxigênio total no banho está diretamente relacionado com o número de inclusões óxidas presentes no aço líquido. O presente trabalho tem como objetivo geral 0 acompanhamento da limpeza inclusionária por meio dos teores de oxigênio total presentes nos aços, para variáveis selecionadas de escória e de processo. A partir das análises químicas de amostras de um aço alto carbono acalmado ao alumínio, foram gerados gráficos com os valores de oxigênio total em função dos parâmetros $\% \mathrm{CaO} / \% \mathrm{Al}_{2} \mathrm{O}_{3}, \% \mathrm{FeO}+\% \mathrm{MnO}$, basicidade binária, tempo de flotação na torre do lingotamento contínuo e pick-up de nitrogênio; seguido da análise dos resultados obtidos e sua comparação com os dados da literatura. Pode-se então concluir que, para as análises anteriormente citadas, o aço apresentou resultados coerentes com a literatura para todas as análises.

Palavras-chave: Escórias; Composição química; Oxigênio total; Flotação.

\section{ANALYSIS OF THE INFLUENCE OF SECONDARY REFINING SLAGS AND PROCESS PARAMETERS IN INCLUSIONS CLEANLINESS OF STEELS \\ Abstract}

The slag utilized in the secondary refining must possess adequate properties so that assist in the control of the inclusions of steel. With this in view, there are various parameters that must be taken in account to obtain an optimal slag that will go assist in the cleaning of the steel. As a technical for measuring, the total oxygen content in the bath is directly related with the number of the oxides inclusions presents in the liquid steel. The present work aims as general objective the accompaniment of the cleaning of the inclusions by means of the levels of the total oxygen presents in steels, for variables selected of the slag and of process. From chemistry analysis of the samples of the a steel high carbon aluminum killed, were generated graphics with the values of the total oxygen in function of the parameters $\% \mathrm{CaO} / \% \mathrm{Al}_{2} \mathrm{O}_{3}$, $\% \mathrm{FeO}+\% \mathrm{MnO}, \% \mathrm{CaO} / \% \mathrm{SiO}_{2}$, time of flotation in the continuous casting turret and pick-up of nitrogen; followed by analysis of the results obtained and comparison with data of the literature. Can be concluded, considering the analysis previously mentioned, steel has shown results consistent with the literature for all analysis.

Keywords: Slag; Chemistry composition; Total oxygen; Flotation.

Graduanda em Engenharia Metalúrgica, Laboratório de Siderurgia (Lasid), Depto. de Metalurgia, UFRGS, Porto Alegre, RS, Brasil.

2 Dr., Laboratório de Siderurgia (Lasid), Depto. de Metalurgia, UFRGS, Porto Alegre, RS, Brasil.

3 Dr.-Ing., Laboratório de Siderurgia (Lasid), Depto. de Metalurgia, UFRGS, Porto Alegre, RS, Brasil.

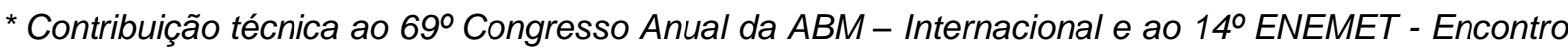
Nacional de Estudantes de Engenharia Metalúrgica, de Materiais e de Minas, 21 a 25 de julho de 2014, São Paulo, SP, Brasil.
} 


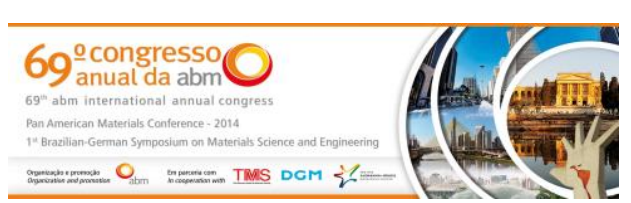

secundário. A composição química empregada em cada escória é empírica e sua eficácia será analisada nos resultados. A tabela 2 apresenta a composição química bruta das escórias do processo.

Tabela 2 - Faixa de composição química bruta da escória do aço SAE 52100 em \% mássica.

\begin{tabular}{|c|c|c|c|c|c|c|}
\hline$(\%)$ & $\mathrm{CaO}$ & $\mathrm{SiO}_{2}$ & $\mathrm{Al}_{2} \mathrm{O}_{3}$ & $\mathrm{MgO}$ & $\mathrm{FeO}$ & $\mathrm{MnO}$ \\
\hline Mínimo & 42,04 & 8,65 & 15,43 & 6,28 & 0,31 & 0,07 \\
\hline Máximo & 57,22 & 20,04 & 27,95 & 20,70 & 6,95 & 0,39 \\
\hline
\end{tabular}

\subsection{Abordagem Experimental}

A coleta das amostras do material e suas respectivas análises foram realizadas em uma planta siderúrgica. Os dados obtidos foram armazenados em um banco de dados históricos ao longo de um ano, para posterior análise das variáveis e comparação com a literatura.

As amostras de aço das barras laminadas foram submetidas à análise de oxigênio total em um Determinador Simultâneo de Nitrogênio e Oxigênio. As amostras de escória tiveram sua composição química definida via espectrometria por fluorescência de Raios-X (XRF). Maiores informações sobre a técnica de análise podem ser encontradas no trabalho de Reis [2]. As composições químicas da escória foram utilizadas para a análise das principais variáveis de escória que influenciam na absorção das inclusões pela escória. Devido ao grande número de corridas representadas, foi utilizada uma análise estatística a fim de se obter o maior número de corridas correspondentes às variáveis analisadas, onde se puderam encontrar as faixas ideais de composição de escória. Por sua vez foram fixadas e comparadas com os dados iniciais. Foram plotados os valores de oxigênio total, $\% \mathrm{CaO} / \% \mathrm{Al}_{2} \mathrm{O}_{3}, \% \mathrm{FeO}+\% \mathrm{MnO}, \% \mathrm{CaO} / \% \mathrm{SiO}_{2}$, tempo de flotação na torre do lingotamento contínuo e pick-up de nitrogênio, em gráficos numa planilha Excel, a fim de se observar a linha de tendência das relações das variáveis com os valores de oxigênio total.

\section{RESULTADOS E DISCUSSÃO}

Os resultados das análises possibilitaram um maior entendimento dos fenômenos físicos e químicos que ocorrem na escória, a fim de se obter um padrão ótimo de escória, propícia à remoção de inclusões para o aço analisado.

\subsection{Influência da Porcentagem de $\mathrm{CaO} / \mathrm{Al}_{2} \mathrm{O}_{3}$ Sobre o Teor de Oxigênio Total do Aço SAE 52100}

Quatro tipos de análises foram feitas para analisar a influência da porcentagem de $\mathrm{CaO} / \mathrm{Al}_{2} \mathrm{O}_{3}$ na escória sobre o valor de oxigênio total.

A figura 1 apresenta o gráfico onde estão presentes os dados de vinte e oito corridas que foram analisados para a primeira análise, onde se fixou o teor de $\mathrm{MgO}$ na escória entre 6 a 8\%. Obteve-se então uma média de 8,71 ppm de oxigênio total e uma baixa tendência do valor de oxigênio total.

A figura 2 apresenta o gráfico onde os dados de oito corridas foram analisados para a segunda análise, onde se manteve fixo o teor de $\mathrm{MgO}$ na escória entre 6 a 8\% e fixou-se apenas os valores de basicidade binária maiores que 4. Obteve-se então

\footnotetext{
* Contribuição técnica ao $69^{\circ}$ Congresso Anual da ABM - Internacional e ao 14ํㅡㄹ ENEMET - Encontro Nacional de Estudantes de Engenharia Metalúrgica, de Materiais e de Minas, 21 a 25 de julho de 2014, São Paulo, SP, Brasil.
} 


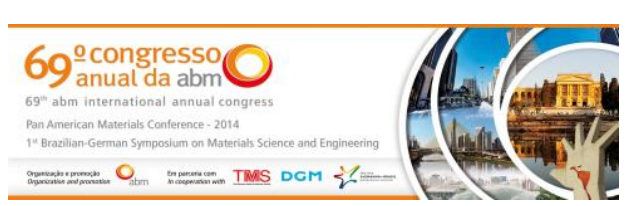

uma média de 8,50 ppm de oxigênio total e uma elevada tendência do valor de oxigênio total.

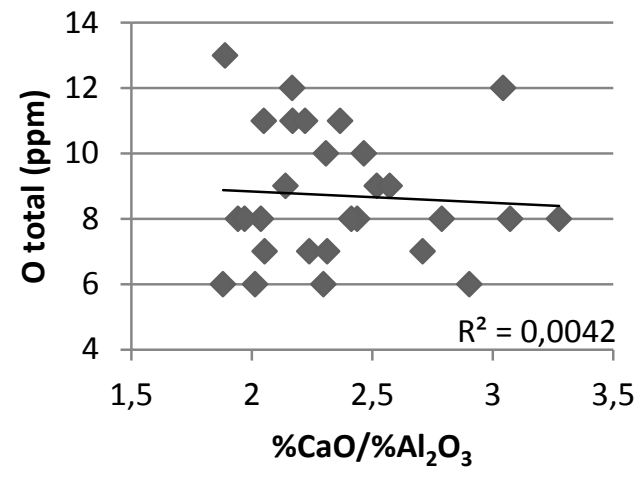

Figura 1 - Evolução do teor de oxigênio total de acordo com o aumento do teor de $\mathrm{CaO} / \mathrm{Al}_{2} \mathrm{O}_{3}$, com $\mathrm{MgO}$ fixo na faixa entre 6 a $8 \%$.

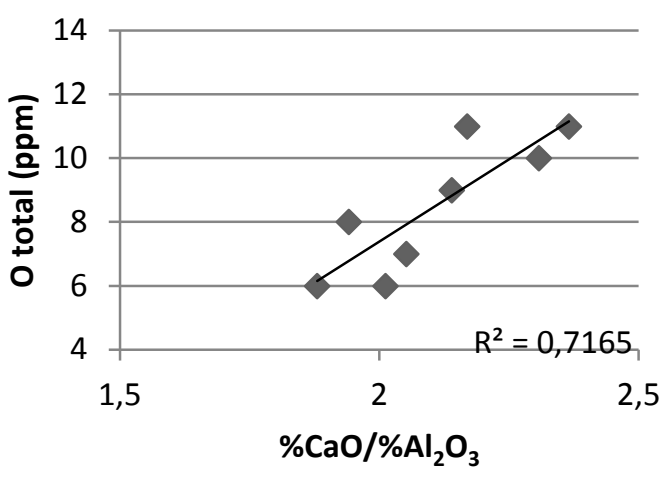

Figura 2 - Evolução do teor de oxigênio total de acordo com o aumento do teor de $\mathrm{CaO} / \mathrm{Al}_{2} \mathrm{O}_{3}$, com $\mathrm{MgO}$ fixo na faixa entre 6 a $8 \%$ e basicidade binária maior que 4 .

A figura 3 apresenta o gráfico onde foram analisados os dados de cinquenta e oito corridas para a terceira análise, onde se fixou o teor de $\mathrm{MgO}$ na escória entre 8 a $12 \%$. Obteve-se então uma média de 9,05 ppm de oxigênio total e uma baixa tendência do valor de oxigênio total.

A figura 4 apresenta o gráfico onde foram analisados os dados de oito corridas para a quarta análise, onde se manteve fixo o teor de $\mathrm{MgO}$ na escória entre 8 a $12 \%$ e fixaram-se apenas os valores de basicidade binária maiores que 4. Obteve-se então uma média de 9,00 ppm de oxigênio total e uma tendência elevada do valor de oxigênio total.

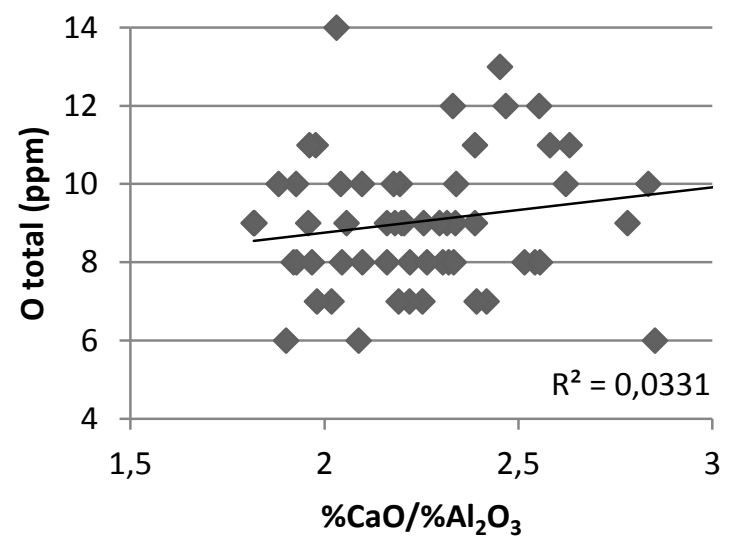

Figura 3 - Evolução do teor de oxigênio total de acordo com o aumento do teor de $\mathrm{CaO} / \mathrm{Al}_{2} \mathrm{O}_{3}$, com $\mathrm{MgO}$ fixo na faixa de 8 a $12 \%$.

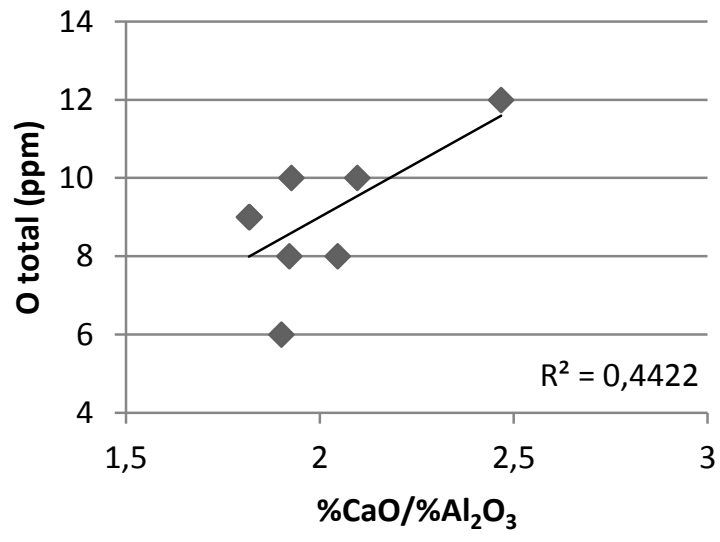

Figura 4 - Evolução do teor de oxigênio total de acordo com o aumento do teor de $\mathrm{CaO} / \mathrm{Al}_{2} \mathrm{O}_{3}$, com $\mathrm{MgO}$ fixo na faixa de 8 a $12 \%$ e basicidade binária maior que 4 .

Sabe-se com base na literatura [3], que a capacidade da escória de absorver inclusões esta diretamente relacionada com a fluidez desta escória.

\footnotetext{
* Contribuição técnica ao 69ำ Congresso Anual da ABM - Internacional e ao 14ํㅡㄹ ENEMET - Encontro Nacional de Estudantes de Engenharia Metalúrgica, de Materiais e de Minas, 21 a 25 de julho de 2014, São Paulo, SP, Brasil.
} 


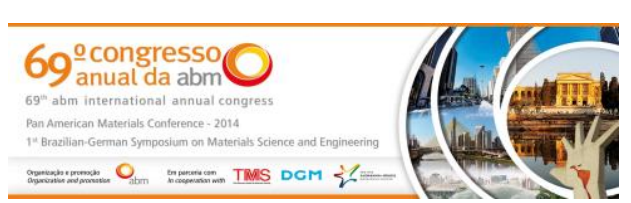

Segundo os estudos realizados por Ma [5] e por Yoon [3] para os aços rolamento, o aumento do índice de basicidade binária leva a uma diminuição dos teores de oxigênio total. Ao analisar-se a figura 5 , sem que nenhuma variável tivesse sido fixada, observa-se a tendência descrita na literatura. Porém, ao analisar-se a figura 6 observa-se o inverso do esperado, e na figura 7 a tendência esperada volta a aparecer. Analisando-se as faixas dos teores de alumina, temos que, na figura 5 abrangem-se todas as faixas de alumina, sem que se tenha fixado quaisquer valores; na figura 6 essa faixa é de 20 a 23\% e na figura 7 tem-se a faixa de 23 a $26 \%$. O que explica o comportamento dos gráficos obtidos, onde a figura 5 nos dá a tendência esperada devido a media de alumina inserida em todas as corridas analisadas; a figura 6 nos dá o inverso do esperado devido ao baixo teor de alumina inserido, o que não possibilita uma fluidez adequada da escória, elevando-se a fração de fase sólida com o aumento da basicidade; e na figura 7 pode-se observar claramente o resultado esperado de acordo com a literatura, devido ao elevado teor de alumina inserido e baixo teor de $\mathrm{MgO}$ controlado, deixando a escória próxima a saturação do $\mathrm{MgO}$ e fluida, devido ao elevado teor de alumina adicionado, o que possibilita uma maior remoção das inclusões aluminosas resultando num baixo teor de oxigênio total.

\subsection{Influência da Porcentagem de FeO + MnO Sobre o Teor de Oxigênio total do Aço SAE 52100}

Três tipos de análises foram feitas para analisar a influência da porcentagem de $\mathrm{FeO}+\mathrm{MnO}$ sobre o valor de oxigênio total.

A figura 8 apresenta o gráfico onde estão presentes os dados de cento e três corridas que foram analisados para a primeira análise, onde não se fixou nenhum teor de nenhuma variável, a fim de se observar a influência que estas causam na análise. Obteve-se então uma média de 8,84 ppm de oxigênio total e uma baixa tendência do valor de oxigênio total.

A figura 9 apresenta o gráfico onde os dados de seis corridas foram analisados para a segunda análise, onde se fixou o teor de MgO na escória entre 6 a 8\%, e teor de alumina de 23 a $26 \%$, e manteve-se apenas os valores de basicidade binária menores que 4. Obteve-se então uma média de 9,83 ppm de oxigênio total e uma tendência do valor de oxigênio total superior à analisada anteriormente.

A figura 10 apresenta o gráfico onde foram analisados os dados de quatro corridas para a terceira análise, onde se fixou o teor de $\mathrm{MgO}$ na escória entre 6 a $8 \%$, e teor de alumina de 23 a $26 \%$, e manteve-se apenas os valores de basicidade binária maiores que 4. Obteve-se então uma média de 9,25 ppm de oxigênio total e uma tendência do valor de oxigênio total superior às analisadas anteriormente.

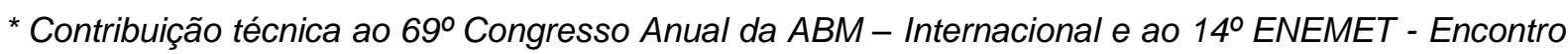
Nacional de Estudantes de Engenharia Metalúrgica, de Materiais e de Minas, 21 a 25 de julho de 2014, São Paulo, SP, Brasil.
} 


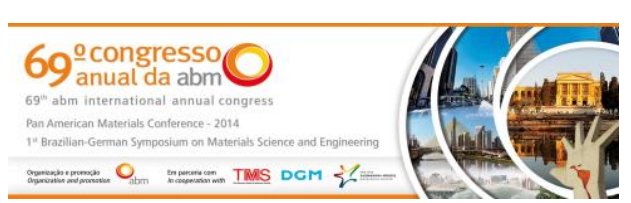

fixando-se os melhores padrões de escória (teor de alumina entre 23 e $26 \%$ e teor de $\mathrm{MgO}$ entre 6 e 8\%) e variando-se a basicidade, encontrou-se uma melhor tendência de acordo com a literatura e, se conseguiu baixar a media de oxigênio total encontrada, que era de 9,83 na figura 9 baixando para 9,25 na figura 10 .

\subsection{Influência do Tempo de Flotação das Inclusões na Torre do Lingotamento Contínuo Sobre o Teor de Oxigênio Total do Aço SAE 52100}

Dois tipos de análises foram realizados para analisar a influência do tempo de flotação das inclusões na torre do lingotamento contínuo sobre o valor de oxigênio total.

A figura 11 apresenta o gráfico onde estão presentes os dados de cento e uma corridas que foram analisados para a primeira análise, onde não se fixou nenhum teor de nenhuma variável, a fim de se observar a influência que estas causam na análise. Obteve-se então uma média de 8,84 ppm de oxigênio total e uma baixa tendência do valor de oxigênio total.

A figura 12 apresenta o gráfico onde os dados de dez corridas foram analisados para a segunda análise, onde se fixou o teor de $\mathrm{MgO}$ na escória entre 6 a $8 \%$, e teor de alumina de 23 a 26\%. Obteve-se então uma média de 9,6 ppm de oxigênio total e uma tendência mais visível do valor de oxigênio total comparada à analisada anteriormente.

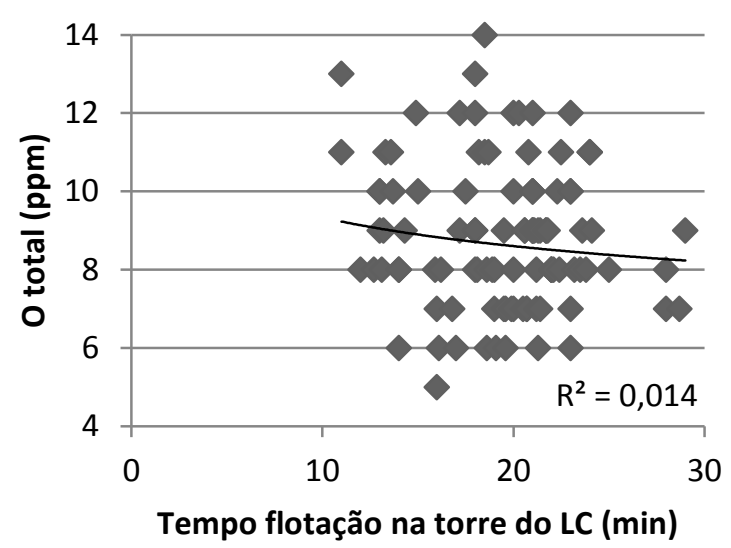

Figura 11 - Evolução do teor de oxigênio total de acordo com o aumento do tempo de flotação na torre do lingotamento contínuo.

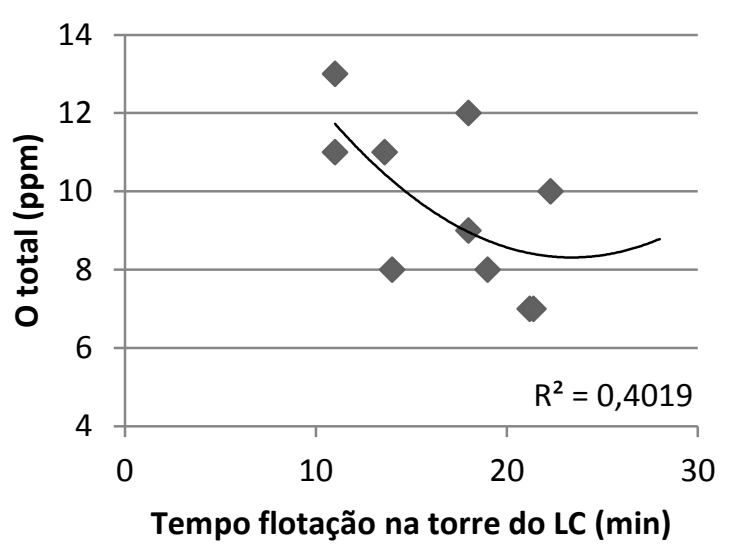

Figura 12 - Evolução do teor de oxigênio total de acordo com o aumento do tempo de flotação na torre do lingotamento contínuo, com $\mathrm{MgO}$ fixo na faixa de 6 a $8 \%$ e alumina fixa na faixa de 23 a $26 \%$.

A agitação da panela no processo de refino promove o crescimento e a remoção das inclusões. Algumas reações metalúrgicas requerem uma forte interação entre metal e escória, enquanto que outras requerem uma mistura mais suave na interface metal/escória além de uma manutenção da camada de escória ininterrupta, como é o caso da remoção de inclusões. As operações de transferência do aço, da panela para o distribuidor e do distribuidor para o molde são muito importantes para a limpeza inclusionária dos aços. A presença de inclusões causa problemas como o entupimento da válvula (clogging), o que gera defeitos no produto final [4].

\footnotetext{
* Contribuição técnica ao $69^{\circ}$ Congresso Anual da ABM - Internacional e ao 14ํㅡㄹ ENEMET - Encontro Nacional de Estudantes de Engenharia Metalúrgica, de Materiais e de Minas, 21 a 25 de julho de 2014, São Paulo, SP, Brasil.
} 


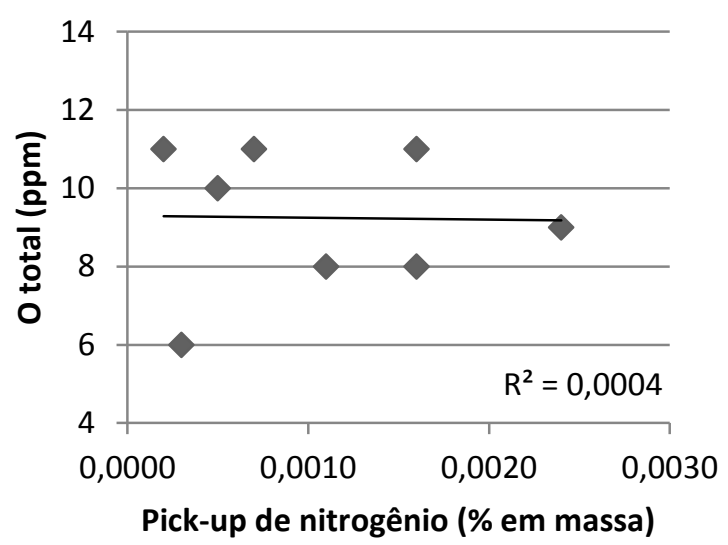

Figura 14 - Evolução do teor de oxigênio total de acordo com o aumento do teor de pick-up de nitrogênio, com alumina fixa na faixa de 20 a $23 \%$.

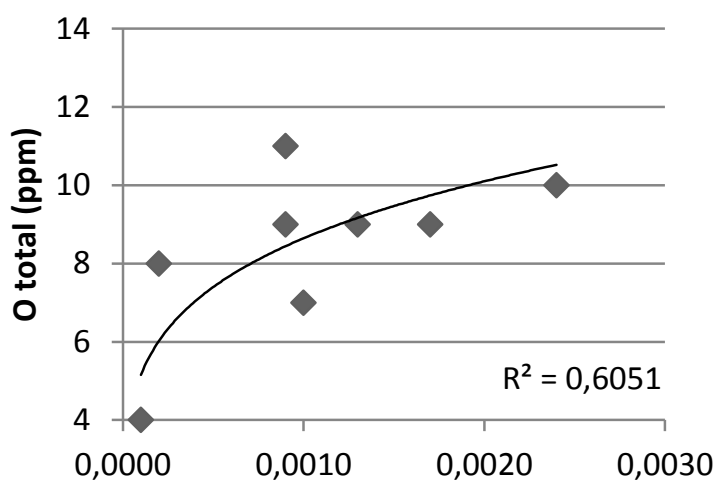

Pick-up de nitrogênio (\% em massa)

Figura 15 - Evolução do teor de oxigênio total de acordo com o aumento do teor de pick-up de nitrogênio, com alumina fixa na faixa de 23 a $26 \%$.

O uso do tubo longo no lingotamento contínuo tem se mostrado uma das práticas mais eficientes na limpeza inclusionária dos aços. Porém, manter a total vedação entre a panela e o distribuidor é uma tarefa praticamente impossível. A interação do aço com o ar atmosférico resulta na reoxidação do mesmo, o uso da análise do pickup de nitrogênio serve como uma medida indireta desta reoxidação [6].

O pick-up de nitrogênio nada mais é do que a medida da diferença entre o nitrogênio dissolvido entre provas do aço em etapas distintas do processo [1].

No estudo feito por Melville [6], foi provado que há um aumento do teor de oxigênio total, conforme é elevado o teor de pick-up de nitrogênio. Ele também mostra que a falta de limpeza do aço devido ao excesso do pick-up de nitrogênio é relativamente pequena se comparada à outras variáveis, tendo estipulado um máximo de 12 ppm para o pick-up de nitrogênio.

No estudo efetuado, foram retiradas as corridas que apresentaram variação negativa de nitrogênio. Sem fixar os valores de alumina, observa-se na figura 13 uma leve tendência a um aumento no teor de oxigênio total. Ao se analisar as figuras 14 e 15, onde foram fixados os teores de alumina, observa-se que, quanto maior for o teor de alumina na escória, maior será o teor de oxigênio total conforme o aumento do pickup de nitrogênio. Tal efeito se deve ao fato de que um alto teor de alumina resulta em uma escória mais líquida, o que favorece mais facilmente a exposição do banho ao ar. Tendo assim, uma tendência positiva do aumento de oxigênio total, o que é de se esperar, já que, após a desoxidação, o baixo nível de oxigênio no banho permite rápida absorção de ar. A este comportamento se atribui à formação de mais inclusões no aço, produtos de reoxidação já mencionados anteriormente. Com base nos dados analisados, se observa uma tendência entre maior pick-up de nitrogênio e maior teor de oxigênio total, como descrito na literatura.

\section{CONCLUSÕES}

A partir das análises efetuadas e dos resultados obtidos, pôde-se fazer uma série de considerações a respeito das variáveis de escória e de processo adotadas neste estudo:

i. A melhor eficiência para a absorção de inclusões da escória se dá com baixos teores de $\mathrm{MgO}$ e elevados teores de $\mathrm{Al}_{2} \mathrm{O}_{3}$.

\footnotetext{
* Contribuição técnica ao 69 Congresso Anual da ABM - Internacional e ao 14ํㅡㄹ ENEMET - Encontro Nacional de Estudantes de Engenharia Metalúrgica, de Materiais e de Minas, 21 a 25 de julho de 2014, São Paulo, SP, Brasil.
} 
ii. Altas basicidades binárias correspondem a uma elevada viscosidade da escória, porém, altos teores de alumina inibem este efeito, sendo observados melhores resultados para basicidades em torno de 4.

iii. Elevados teores de $\mathrm{CaO} / \mathrm{Al}_{2} \mathrm{O}_{3}$ fornecem 0 cálcio necessário para 0 tratamento das inclusões, porém, também resultam em uma escória de fase sólida elevada, o que implica em uma baixa capacidade de absorção de inclusões. Sendo assim, torna-se necessário o controle do teor de $\mathrm{CaO}$ para que se trabalhe com uma escória rica, tanto em fase líquida quanto em cálcio.

iv. Altos teores de $\mathrm{FeO}$ e $\mathrm{MnO}$ na escória resultam na reoxidação do banho e, consequentemente, um aumento do número de inclusões de alumina presentes no aço.

v. A agitação do banho feita por gases inertes auxilia na remoção das inclusões, sendo necessário o controle do tempo para que haja a devida flotação, e não se perca muita temperatura expondo o banho ao ar.

vi. Elevados teores de pick-up de nitrogênio significam uma alta reoxidação do banho, o que implica em maiores quantidades de inclusões. Sendo a análise do pick-up a menos impactante com relação à limpeza inclusionária dos aços, devido ao enorme controle e baixos teores de pickup de nitrogênio observados.

Como consideração final tem-se que, o conhecimento das variáveis de escória e de processo é de suma importância para que seja feito um controle inclusionário de qualidade. O completo entendimento dos fenômenos metalúrgicos envolvidos na produção de aços especiais, assim como a adequação dessas variáveis possibilita um produto final de qualidade, algo extremamente valorizado nos dias atuais.

\section{Agradecimentos}

Ao Professor Dr. Wagner Viana Bielefeldt pela orientação, conselhos e amizade.

Ao Professor Dr-Ing. Antônio C. F. Vilela pela confiança.

Ao Laboratório de Siderurgia da UFRGS pelos recursos e pela bolsa de iniciação científica.

Ao Dr. Eng. Julio Aníbal Morales Pereira pela amizade, apoio e conselhos.

Aos colegas e amigos do Laboratório de Siderurgia pelo cooperativismo, em destaque à Caroline Almeida pelo companheirismo e amizade.

\section{REFERÊNCIAS}

1 Bartosiaki BG. Caracterização de inclusões não metálicas de óxidos no aço SAE 52100. Trabalho de Diplomação, Escola de engenharia, UFRGS, 2013.

2 Reis $\mathrm{BH}$. Estudo sobre a capacidade de absorção de inclusões pela escória de refino secundário na fabricação de aços. Trabalho de Diplomação, Escola de engenharia, UFRGS, 2013.

3 Yoon BH, Heo KH, Kim JS.; Sohn, H.S. Improvement of Steel cleanliness by controlling slag composition. Ironmaking and Steelmaking, v.29, n.3, p.215-218, 2002.

4 Zhang L, Thomas BG. State of the Art in Evaluation and Control of Steel Cleanliness. ISIJ International, 2002; 43(3): 271-291, 2002.

5 Ma JW, Bao PY, Wang M, Zhao WD. Influence of slag composition on bearing steel cleanness. Ironmaking and Steelmaking, 2014; 4(1): 26-30.

6 Melville DS, Brinkmeyer L. Evaluating Steelmaking and Casting Practices Which Affect Quality, 78th Steelmaking Conf. Proc., ISS, Warrendale, PA, (1995), 563.

\footnotetext{
* Contribuição técnica ao $69^{\circ}$ Congresso Anual da ABM - Internacional e ao 14ํㅡㄹ ENEMET - Encontro Nacional de Estudantes de Engenharia Metalúrgica, de Materiais e de Minas, 21 a 25 de julho de 2014, São Paulo, SP, Brasil.
} 